\title{
Perfil epidemiológico dos casos e óbitos por COVID-19 nos estados da região nordeste
}

\author{
Epidemiological profile of cases and deaths by COVID-19 in the states of the northeast \\ region
}
Perfil epidemiológico de casos y muertes por COVID-19 en los estados de la región noreste

Gabriela Romão de Almeida Carvalho Santos ${ }^{1 *}$, Letícia Seara Gama1, Adriele de Santana dos Santos $^{1}$, Victória Almeida Santos Nascimento ${ }^{1}$, Ruama de Souza Nogueira ${ }^{1}$, Brenda dos Anjos Tosta da Silva ${ }^{1}$, Cintia Maria Magalhães Oliveira de Araujo², Mayana de Almeida Sento-Sé Passos ${ }^{3}$, Aglaya Oliveira Lima Cordeiro de Almeida'.

\begin{abstract}
RESUMO
Objetivo: Este estudo visa identificar o perfil epidemiológico dos casos confirmados e de óbitos pela Doença do Coronavírus (COVID-19) nos estados da região Nordeste do Brasil. Métodos: Trata-se de um estudo ecológico de série temporal, tendo seus dados apresentados sob forma de frequência absoluta e relativa, adotando como variáveis: sexo, faixa etária e região, no qual os dados coletados foram do período de 15 de março a 23 de abril de 2020 a partir de dados secundários extraídos dos boletins epidemiológicos de cada estado do Nordeste do Brasil. Resultados: O estado com maior prevalência em números de casos e óbitos foi o Pernambuco, seguido do Ceará e Bahia. Em três estados observou-se divisão igualitária entre sexos nos números de casos, nos demais, o sexo feminino predominou, sendo a faixa etária mais acometida entre 2049 anos. Referente aos óbitos por sexo, o masculino foi mais acometido, entre 20-49 anos observou-se mais mortes. Conclusão: Por se tratar de um estudo de acompanhamento, a elucidação e confirmação das conclusões advindas dos vigentes resultados, só serão possíveis com o avanço dos conhecimentos.
\end{abstract}

Palavras-chave: Infecções por coronavírus, Perfil de saúde, Brasil.

\begin{abstract}
Objective: This study aims to identify the epidemiological profile of confirmed cases and deaths by Coronavirus Disease (COVID-19) in the states of the Northeast region of Brazil. Methods: This is an ecological time series study, with its data presented in the form of absolute and relative frequency, adopting as variables: sex, age group and region, in which the data collected were from the period from March 15 to 23 April 2020 from secondary data extracted from the epidemiological bulletins of each state in the Northeast region of Brazil. Results: The states with the highest prevalence in numbers confirmed cases and deaths was Pernambuco, followed by Ceará and Bahia. An equal division between the sexes was observed in three states in numbers of confirmed cases, in others states the male sex prevailed, and the hardest hit age group was between 20 49 years old. Sex-related deaths, the male sex was the most affected, between 20-49 years more deaths was observed. Conclusion: It is worth noting that, as it is a follow-up study, the elucidation and confirmation of the conclusions from these current results, will only be possible with the advance of knowledge about the theme.
\end{abstract}

Keywords: Coronavirus infections, Health profile, Brazil.

\footnotetext{
1 Universidade Salvador (UNIFACS), Salvador - BA. *E-mail: gabrielaromaoalmeida98@gmail.com

${ }^{2}$ Hospital Naval de Salvador, Salvador - BA.

${ }^{3}$ Unidade de Pronto Atendimento (UPA), Salvador - BA.
} 


\section{RESUMEN}

Objetivo: Este estudio tiene como objetivo identificar el perfil epidemiológico de los casos confirmados y las muertes por Enfermedad del coronavirus (COVID-19) en los estados de la región Noreste de Brasil. Métodos: Es un estudio ecológico de series temporales, teniendo sus datos presentados en forma de frecuencia absoluta y relativa, adoptando como variables: género, grupo de edad y región, en el que los datos recogidos fueron del 15 de marzo al 23 de abril de 2020 a partir de datos secundarios extraídos de los boletines epidemiológicos de cada estado de la región noreste de Brasil. Resultados: El estado con mayor prevalencia en número de casos y muertes fue Pernambuco, seguido de Ceará y Bahía. En tres estados se observó una división igual entre sexos en el número de casos, en los demás, predominó el sexo femenino, siendo el grupo de edad más afectado entre 20-49 años. Sobre muertes por sexo, los hombres fueron más afectados, entre 20-49 años se observaron más muertes. Conclusión: Como se trata de un estudio de seguimiento, la aclaración y confirmación de las conclusiones de los resultados actuales solo será posible con el avance del conocimiento.

Palabras clave: Infecciones por coronavirus, Perfil de salud, Brasil.

\section{INTRODUÇÃO}

A atual pandemia por Coronavírus teve início no final de 2019, em Wuhan na China, onde o aparecimento de uma pneumonia de origem etiológica desconhecida levou a descoberta de uma nova linhagem do Coronavírus, o SARS-CoV-2 (LANA RM, et al., 2020; BELASCO AGS e FONSECA CD, 2020).

Esse novo vírus pertence à família coronaviridae e foi originado a partir de recombinações virais que permitiram infectar, além dos animais, seres humanos também. Como se trata de um RNA vírus, há uma predisposição maior a mutações e uma transmissibilidade elevada, além de uma alta virulência (GORBALENYA AE, et al., 2020; LU R, et al., 2019; LI Q, et al., 2020; SCHATZMAYR HG, 2001).

No Brasil, segundo dados do Ministério da Saúde, o primeiro caso da COVID-19 surgiu no dia 26 de fevereiro de 2020, no estado de São Paulo, sendo este o território brasileiro mais afetado pela doença, com aproximadamente 390.000 casos confirmados e cerca de 18.000 óbitos.

Atualmente, o país está próximo da marca de 2.000 .000 de casos confirmados, registrando mais de 75.000 óbitos, sendo assim, o índice de mortalidade/habitante no Brasil atinge um valor de 35,9/100 mil habitantes, enquanto a região Nordeste do país apresenta um índice de mortalidade de 42,9/100 mil habitantes.

Desta forma, a área representa a segunda maior relação de mortalidade por habitante do país, ficando atrás da região Norte, cujo coeficiente de mortalidade equivale a 58/100 mil habitantes, sendo o maior do Brasil (BRASIL, 2020).

O SARS-CoV-2 é responsável pelo desenvolvimento da doença do Coronavírus (COVID-19), que inicia com um quadro de pneumonia e rapidamente pode evoluir para a Síndrome Respiratória Aguda Grave (SRAG) ou até mesmo para complicações mais graves e óbito.

A COVID-19 é transmitida através de secreções respiratórias, tendo um período de incubação entre 1 a 14 dias, afetando a todos os seres humanos, em especial os idosos e pessoas com comorbidades. Os sintomas podem ser desde uma febre, tosse seca, dispneia até sintomas mais graves causados por uma desordem sistêmica e respiratória, os quais estão associados a uma maior taxa de mortalidade (CHEN N, et al., 2020; GUO YR, et al., 2020; ROTHAN HÁ, et al., 2020).

Em 30 de janeiro de 2020 a Organização Mundial de Saúde (OMS) declarou uma Emergência de Saúde Pública em decorrência do surto causado pela COVID-19, e em 11 de março a OMS considerou tal surto como uma pandemia. De caráter extremamente preocupante, já foram, de acordo com a OMS e Ministério da Saúde, mais de 13.150 .645 casos e 574.464 mortes confirmados por COVID-19 no mundo (OMS, 2020). 
O Brasil, assim como em todo mundo, tem sua curva crescendo de forma exponencial, sendo registrados 1.966.748 casos confirmados e 75.366 mortes, apresentando-se como uma doença grave. Nesse contexto, com relação a taxa de incidência do Coronavírus, o país apresenta uma taxa equivalente a 935,9/100 mil habitantes, ademais o Nordeste apresenta um índice de 1155,9/100 mil habitantes, obtendo assim uma taxa maior que a média nacional (BRASIL, 2020a; FREITAS ARR, et al., 2020). Considerando-se o objeto de estudo proposto, o presente artigo tem como objetivo identificar o perfil epidemiológico dos casos confirmados e de óbitos pela COVID-19 nos estados da região Nordeste.

\section{MÉTODOS}

Estudo ecológico de série temporal, no qual os dados coletados foram do período de 15 de março a 23 de abril de 2020 a partir de dados secundários extraídos dos boletins epidemiológicos de cada estado da região Nordeste do Brasil.

Tendo como objeto de pesquisa " perfil epidemiológico dos casos e óbitos por covid-19 na região nordeste do Brasil e para nortear a busca de dados foi elaborada a seguinte questão: qual perfil epidemiológico dos casos e óbitos por covid-19 na região nordeste do Brasil?

A população de estudo foi formada por indivíduos de todas as faixas etárias, de ambos os sexos, acometidos pelo COVID-19 no ano de 2020. Os dados foram tabulados considerando-se as seguintes variáveis de cada estado da região Nordeste: 1) Casos confirmados de indivíduos por COVID-19 por sexo; 2) Casos confirmados de indivíduos por COVID-19 por faixa etária; 3) Óbitos de indivíduos por COVID-19 por sexo; 4) Óbitos de indivíduos por COVID-19 por faixa etária; 5) Casos confirmados e óbitos de indivíduos por COVID-19 por região.

Para análise dos dados, foi utilizado o programa Microsoft Excel 2013 e Microsoft Word, sendo os resultados apresentados sob forma de frequências absolutas e relativas a partir de duas tabelas e dois gráficos. Por se tratar de um banco de dados de domínio público, não foi necessária submissão ao comitê de ética em pesquisa.

\section{RESULTADOS}

Para delinear o perfil epidemiológico dos casos confirmados e óbitos por COVID-19 na região nordeste, foram analisados 8.948 casos confirmados e 536 óbitos por esta causa no período de 15 de março a 23 de abril de 2020 quanto às variáveis sexo, faixa etária e estados da região nordeste. No que diz respeito aos estados da região nordeste, Pernambuco possui 2.193 casos confirmados, sendo o estado com maior número de confirmações, seguido do Ceará com 1.989 casos.

O estado da Bahia apresentou 1.962 casos confirmados, o Maranhão 1.205 casos, logo em seguida o estado do Rio Grande do Norte com 754 casos, seguido de Alagoas com 324 casos, a Paraíba com 263 casos, Sergipe com 135 casos e o estado com menor número de casos é o Piauí, com 123 casos confirmados.

Com relação ao número de óbitos por COVID-19 nos estados da região nordeste, Pernambuco permanece em primeiro lugar com 205 óbitos, seguido do Ceará com 111 óbitos. O estado da Bahia apresentou 62 óbitos, o Maranhão 48 óbitos, o Rio Grande do Norte com 38 óbitos, Paraíba com 33 óbitos, Alagoas com 22 óbitos, o Piauí com 9 óbitos e o estado com menor número de óbitos foi Sergipe com 8 óbitos.

Com isso, pôde-se perceber que os números de mortes são proporcionais a quantidade de casos, visto que os estados permanecem na mesma posição ao confrontar casos confirmados e óbitos, com exceção da Paraíba e Alagoas que trocam as posições, assim como Piauí e Sergipe, que inverteram suas posições.

No que diz respeito à distribuição entre os sexos de acordo com o número de casos confirmados, Paraíba, Alagoas e Rio Grande do Norte tiveram uma distribuição praticamente igualitária, atingindo ambos os sexos de forma equivalente. 
Nos demais estados da região Nordeste observou-se que o sexo feminino foi o mais acometido, com exceção ao estado do Piauí, no qual o número de notificações foi mais prevalente no sexo masculino (52,80\%) (Gráfico 1).

Gráfico 1 - Número de casos confirmados da COVID-19 por sexo nas regiões do Nordeste.

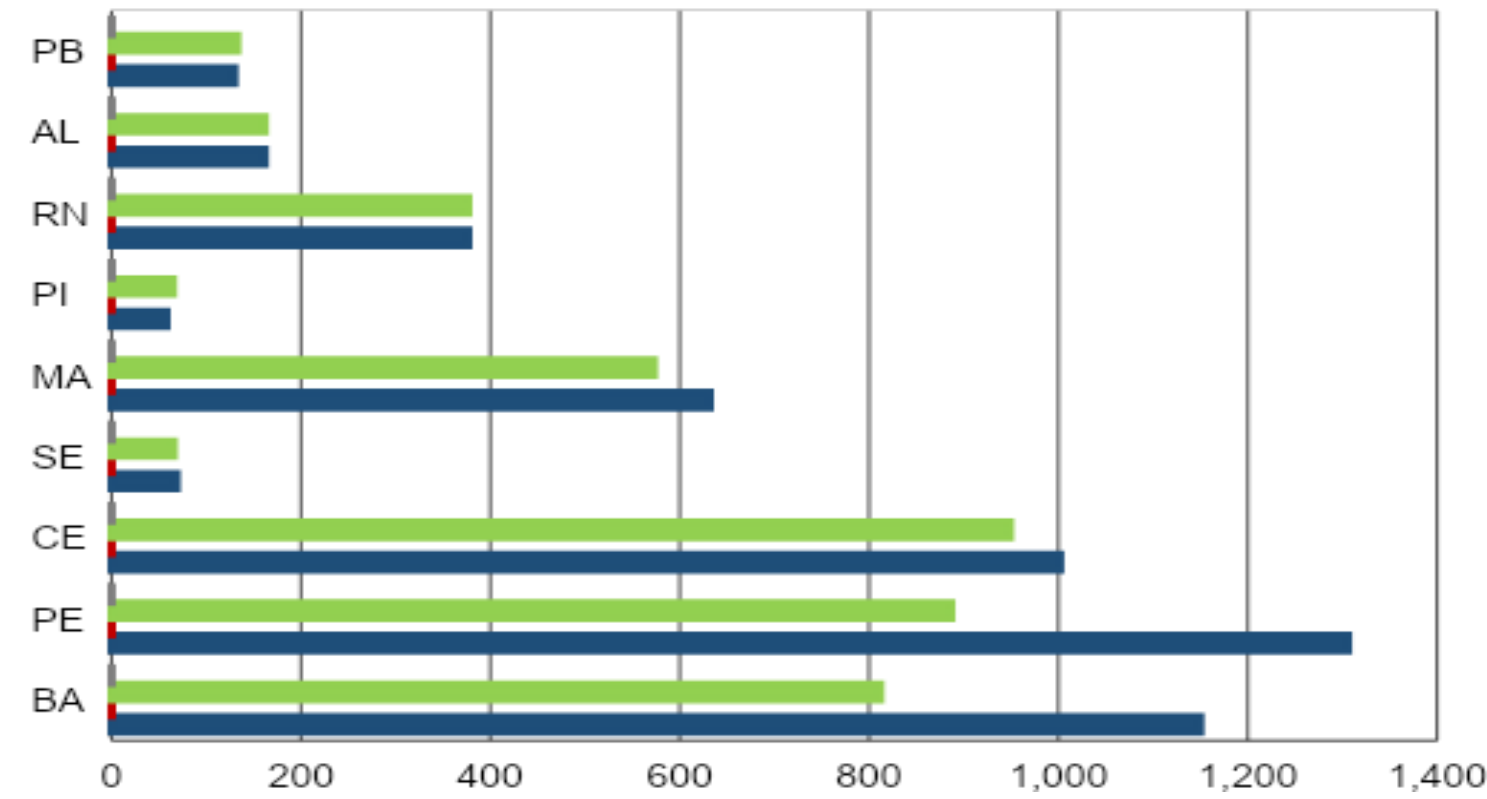

Fonte: Santos GRAC, et al., 2020. Baseado na Secretaria de Saúde, 2020.

Com relação aos dados coletados, em aproximadamente $2.47 \%$ dos casos, a idade não estava presente nas bases de dados. Sendo assim, percebe-se que a faixa etária com maior proporção dos casos confirmados de COVID-19 em todos os estados do Nordeste foi a de 20 a 49 anos, representando uma taxa média de $64.82 \%$ dos casos nessa faixa.

Dentre os casos confirmados, a faixa etária com menor número de casos foi a de 0 a 19 anos, com uma média de $3.98 \%$. Contudo, os boletins epidemiológicos do estado de Ceará não apresentavam informações no mesmo intervalo de faixa etária que os outros estados, sendo este um fator limitante para análise (Tabela 1).

Tabela 1 - Número de casos confirmados da COVID-19 por faixa etária nas regiões do Nordeste.

\begin{tabular}{|c|c|c|c|c|c|c|c|c|c|c|}
\hline \multirow{2}{*}{$\begin{array}{l}\text { Faixa } \\
\text { etária }\end{array}$} & \multicolumn{2}{|c|}{$0-19$} & \multicolumn{2}{|c|}{$20-49$} & \multicolumn{2}{|c|}{$50-69$} & \multicolumn{2}{|c|}{ Acima de 70} & \multicolumn{2}{|c|}{$\begin{array}{c}\text { Não } \\
\text { informado }\end{array}$} \\
\hline & № & $\%$ & № & $\%$ & № & $\%$ & № & $\%$ & № & $\%$ \\
\hline$A L$ & 11 & $3,39 \%$ & 201 & $62,03 \%$ & 80 & $24,69 \%$ & 32 & $9,87 \%$ & 0 & $0 \%$ \\
\hline BA & 102 & $8,50 \%$ & 1185 & $98,75 \%$ & 376 & $31,30 \%$ & 168 & $14,00 \%$ & 131 & $6,70 \%$ \\
\hline CE & 67 & $3,36 \%$ & 1131 & $56,86 \%$ & 528 & $26,54 \%$ & 225 & $11,31 \%$ & 38 & $1,91 \%$ \\
\hline PB & 5 & $1,90 \%$ & 162 & $61,59 \%$ & 58 & $22,05 \%$ & 38 & $14,44 \%$ & 0 & $0 \%$ \\
\hline PE & 119 & $5,42 \%$ & 1295 & $59,05 \%$ & 563 & $25,67 \%$ & 276 & $12,58 \%$ & 0 & $0 \%$ \\
\hline PI & 4 & $3 \%$ & 67 & $54,47 \%$ & 39 & $31,70 \%$ & 13 & $10,56 \%$ & 0 & $0 \%$ \\
\hline MA & 22 & $1,82 \%$ & 796 & $66,05 \%$ & 285 & $23,65 \%$ & 97 & $8,04 \%$ & 9 & $0,74 \%$ \\
\hline RN & 25 & $3,35 \%$ & 476 & $63,89 \%$ & 187 & $25,10 \%$ & 62 & $8,32 \%$ & 4 & $0,54 \%$ \\
\hline SE & 6 & $5,12 \%$ & 71 & $60,68 \%$ & 28 & $23,93 \%$ & 10 & $8,54 \%$ & 0 & $0 \%$ \\
\hline
\end{tabular}

Fonte: Santos GRAC, et al., 2020. Baseado na Secretaria de Saúde, 2020. 
Tabela 2 - Número de óbitos por COVID-19 de acordo com a faixa etária nas regiões do Nordeste.

\begin{tabular}{|c|c|c|c|c|c|c|c|c|c|c|}
\hline \multirow{2}{*}{$\begin{array}{l}\text { Faixa } \\
\text { etária }\end{array}$} & \multicolumn{2}{|c|}{$0-19$} & \multicolumn{2}{|c|}{$20-49$} & \multicolumn{2}{|c|}{$50-69$} & \multicolumn{2}{|c|}{ Acima de 70} & \multicolumn{2}{|c|}{$\begin{array}{c}\text { Não } \\
\text { informado }\end{array}$} \\
\hline & № & $\%$ & № & $\%$ & № & $\%$ & № & $\%$ & № & $\%$ \\
\hline AL & 11 & $3 \%$ & 201 & $62,00 \%$ & 80 & $24,69 \%$ & 32 & $9,87 \%$ & 0 & $0 \%$ \\
\hline BA & 102 & $6 \%$ & 1185 & $64,71 \%$ & 376 & $20,53 \%$ & 168 & $9,17 \%$ & 0 & 0 \\
\hline CE & 67 & $3,43 \%$ & 1131 & $57,97 \%$ & 528 & $27,06 \%$ & 225 & $11,53 \%$ & 0 & $0 \%$ \\
\hline PB & 5 & $1,88 \%$ & 162 & $61,13 \%$ & 58 & $21,88 \%$ & 38 & $14,33 \%$ & 2 & $0,75 \%$ \\
\hline PE & 119 & $5,28 \%$ & 1295 & $57,47 \%$ & 563 & $24,98 \%$ & 276 & $12,25 \%$ & 0 & $0 \%$ \\
\hline PI & 4 & $3,25 \%$ & 67 & $54,47 \%$ & 39 & $31,70 \%$ & 13 & $10,56 \%$ & 0 & $0 \%$ \\
\hline MA & 22 & $1,88 \%$ & 796 & $66,27 \%$ & 285 & $23,73 \%$ & 97 & $8,07 \%$ & 1 & $0,08 \%$ \\
\hline RN & 2 & $0,27 \%$ & 476 & $65,47 \%$ & 187 & $25,72 \%$ & 62 & $8,52 \%$ & 0 & $0 \%$ \\
\hline SE & 6 & $5,17 \%$ & 71 & $61,20 \%$ & 28 & $24,13 \%$ & 10 & $8,62 \%$ & 1 & $0,86 \%$ \\
\hline
\end{tabular}

Fonte - Santos GRAC, et al., 2020. Baseado na Secretaria de Saúde, 2020.

Relacionado ao número de casos de óbitos por sexo, observou-se que em todos os estados do Nordeste, o sexo masculino foi o mais acometido. Com exceção do estado de Pernambuco, que apresentou uma taxa de óbito maior na população feminina (53.70\%), a qual se enquadra em um modelo diretamente proporcional ao número de casos confirmados, que é composto de forma majoritária por mulheres, discordando do padrão visto nos outros estados, onde, embora houvesse uma concentração menor de casos confirmados no sexo masculino, o óbito foi maior nesse grupo. Além disso, notou-se uma discrepância significativa no estado do Piauí, no qual o sexo masculino teve o maior número de mortes $(77.80 \%)$ comparado ao sexo feminino (22.20\%) (Gráfico 2).

Gráfico 2 - Número de óbitos por COVID-19 de acordo com o sexo nas regiões do Nordeste.

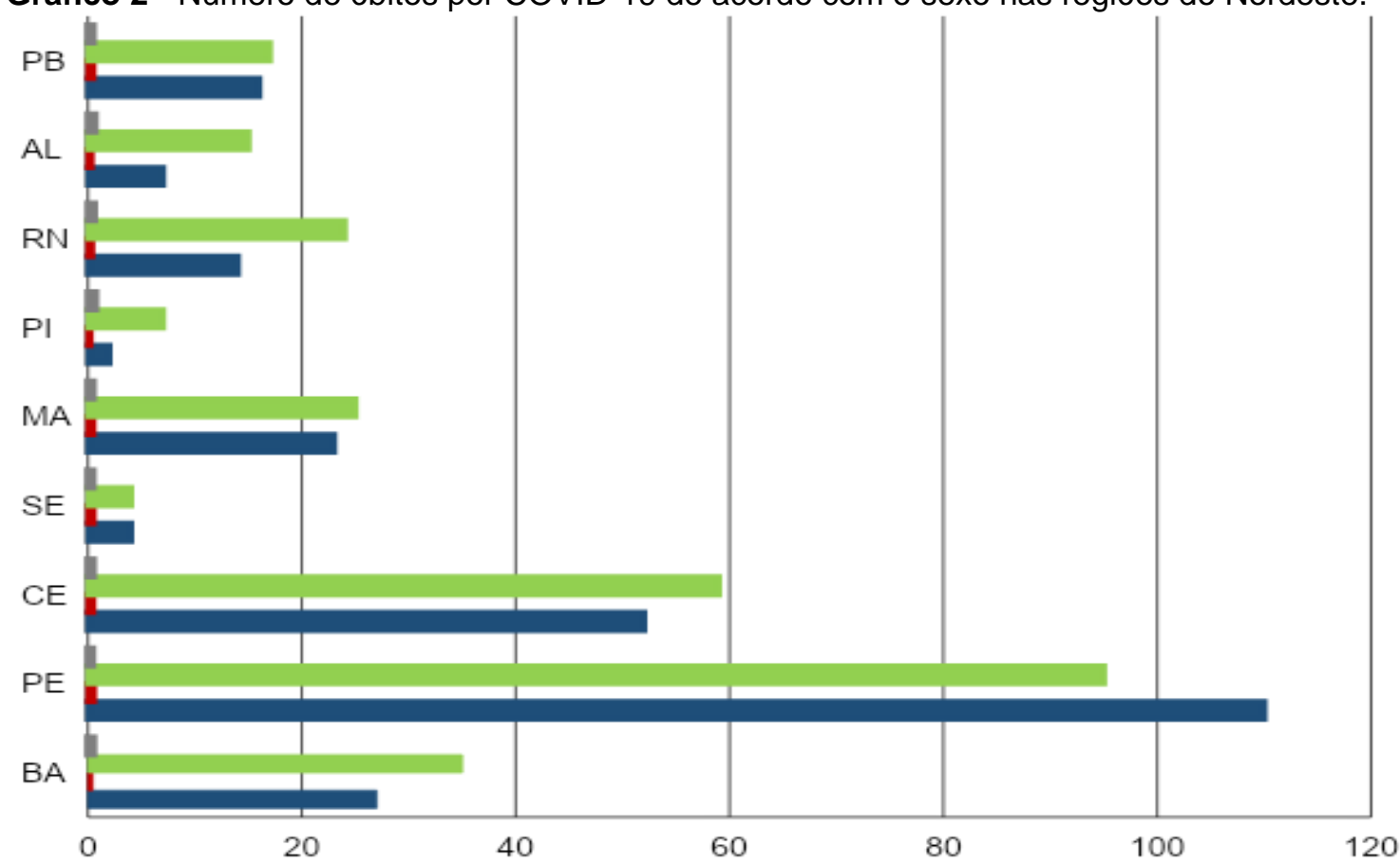

Fonte: Santos GRAC, et al., 2020. Baseado na Secretaria de Saúde, 2020. 
Quando analisados os números de óbitos por faixa etária, ao comparar a faixa etária mais atingida, foi possível interpretar que a faixa mais prevalente foi a de 20 a 49 anos de idade. Além disso, nota-se que, em todas as regiões, a faixa de 10 a 19 anos foi a menos acometida. Em contrapartida, devido à ausência de dados no boletim epidemiológico do estado da Bahia, não foi possível construir uma análise comparativa do perfil etário, associado a isso, o estado do Ceará não informou o número de óbitos referente ao mesmo intervalo de faixa etária dos outros estados, sendo assim os coeficientes restritivos dessa vertente de análise (Tabela 2).

\section{DISCUSSÃO}

Os resultados obtidos no estudo mostraram que em relação aos casos confirmados, o sexo feminino foi o mais afetado, com exceção da Paraíba, Alagoas e Rio Grande do Norte, onde não houve desproporção entre os sexos. Válido ressaltar que Piauí tangenciou o delineamento, mostrando uma prevalência na população masculina. Ainda analisando os casos confirmados, percebe-se que a faixa etária mais atingida foi entre 20 49 anos, sendo o estado da Bahia o mais à frente. Referente aos óbitos por sexo, o grupo masculino foi o mais culminado, ademais, mantendo-se na vertente de análise, a faixa etária de 20-49 anos obteve mais mortes.

Há relatos de vivência clínica que demonstram que muitos pacientes entre 20 a 40 anos de idade estão sendo infectados pelo novo coronavírus. Os dados obtidos no vigente estudo demonstram a mesma prevalência, isso pode ser explicado pelo perfil da população brasileira, a qual é composta, majoritariamente, por jovens adultos (RENTE A, et al., 2020; CRODA J, et al., 2020).

Durante o período analisado, embora Pernambuco apresente um padrão diferente dos demais estados, em relação a taxa de óbitos, a qual foi mais prevalente entre as mulheres, não foi encontrado na literatura, até então, evidências que expliquem esse delineamento controverso, mas é inegável que ainda assim a população masculina tem um papel de destaque quando se trata de óbitos por COVID-19 no Nordeste.

De acordo com Brasil (2020 b), cerca de $77 \%$ dos brasileiros do sexo masculino seguem as orientações de higiene da OMS com relação a prevenção do Coronavírus, e no que diz respeito ao sexo feminino dessa amostra, mais de $85 \%$ adotam as orientações de higiene.

Esses dados permitem interpretar que os homens se infectam mais que as mulheres, porém os boletins epidemiológicos analisados demonstram que a taxa de contaminação por COVID-19 é maior no sexo feminino, induzindo a interpretar que essas medidas de higiene não possuem relação diretamente proporcional ao número de casos. Contudo, levando em consideração que esses dados foram obtidos através de um inquérito telefônico, não é possível afirmar com exatidão se de fato as indicações de higiene estão sendo realizadas de maneira adequada (LIMA DLF, 2020).

Em relação ao número de casos de óbitos por sexo, observou-se que em todos os estados do Nordeste, com exceção de Pernambuco, o sexo masculino foi o mais acometido, o que permite depreender que as mulheres são as mais infectadas, porém os homens morrem mais por Coronavírus.

Vários estudos afirmam que o sexo masculino, em geral, são os mais acometidos por condições severas e crônicas de saúde do que o gênero feminino e também morrem mais do que as mulheres, o que poderia explicar a prevalência de óbitos por COVID-19 nos homens no Nordeste, mesmo com um perfil de infectados predominantemente feminino na região (DIAS FLT, et al., 2020).

É válido ressaltar que, embora o presente estudo apresente uma prevalência das mulheres no número de casos confirmados de COVID-19, dois estudos realizados em Wuhan na China, constataram que o sexo mais acometido foi o masculino. Essa diferença poderia ser explicada por conta do perfil social do Brasil, que é diferente quando comparado a população chinesa, entretanto, um outro estudo realizado no estado do Ceará, contabilizou que o gênero masculino também foi o mais acometido pelo Coronavírus, o que diverge dos dados encontrados na atual pesquisa, uma possível explicação para esse contexto é o método utilizado para análise, que foi através de um questionário online, diferentemente do vigente estudo, que teve como fonte os boletins epidemiológicos (WU C, et al., 2020; LI Q, et al. 2020; CHEN N, et al., 2020). 
Esse mesmo estudo realizado no estado do Ceará, evidenciou que as mulheres que se enquadram na faixa de 20-39 anos e aqueles que possuem um nível elevado de escolaridade mostraram-se mais preocupados com a contaminação pelo Coronavírus. Além disso, essa pesquisa mostrou que, devido aos fatores comportamentais e sociais dos idosos, eles se intitulam como risco médio para contaminação (LIMA DLF, et al., 2020). Dessa forma, tais dados permitem interpretar que a condição social e o baixo nível escolar atuam como fatores de risco para a COVID-19.

Diante desses resultados, é possível inferir que embora a população feminina predomine o número de casos confirmados de COVID-19, a população masculina tem destaque no número de óbitos pela doença. Ademais, não há disponibilidade de literatura suficiente para esclarecer os conflitos levantados durante a discussão dos achados.Segundo o Conselho Federal de Enfermagem, a maioria dos profissionais de enfermagem é do sexo feminino, correspondendo a $87,24 \%$ dos profissionais do Brasil, enquanto o sexo masculino corresponde a $12,76 \%$ do total dos profissionais, evidenciando assim que tem mais mulheres na linha de frente de combate ao Coronavírus, tal fato pode explicar os resultados encontrados no presente estudo, afinal dessa forma há um aumento das chances de contágio, devido a uma maior exposição ao vírus (COFEN, 2011).

De acordo com um estudo publicado, pessoas acima de 50 anos infectadas pelo Coronavírus têm 2.6 vezes mais chances de evoluir para forma grave da doença, além disso, um paciente em estado grave pelo novo vírus ocupa o leito por no mínimo 15 dias. Com base nessas informações e considerando que, em Pernambuco, a população representa $59,05 \%$ de todos os casos confirmados de COVID-19, percebe-se que boa parte pode precisar utilizar os leitos de UTI do estado, os quais compreendem um número de 256, tendo em vista que a probabilidade da progressão da doença nessa faixa etária é elevada. Esses dados permitem inferir que, uma possível explicação para o elevado número de óbitos no estado por COVID-19, seria em decorrência da incapacidade da atenção terciária de Pernambuco fornecer suporte avançado a esse público (MA C, et al., 2020; AMIB, 2020; SESPE, 2020).

Com base nos dados contidos nos boletins epidemiológicos utilizados no vigente estudo, é notório que o estado do Piauí foi o menos afetado no período analisado. Durante a análise a taxa de isolamento social no estado variou entre $40 \%$ a $50 \%$, apresentando um decréscimo de $8,1 \%$ no período final do intervalo em estudo, em contrapartida Pernambuco durante o mesmo espaço de tempo demonstrou uma taxa de isolamento maior que a obtida em Piauí, variando entre $44,8 \%$ a $62 \%$, registrando no final da amostra, um valor de $46,3 \%$, o que permite inferir que mesmo o distanciamento coletivo sendo a medida mais eficaz no controle da COVID-19, a adesão por parte da população não foi um fator determinante para incidência de casos, sendo essa uma variável sem dimensão proporcional nesse caso, tendo em vista que o estado do Piauí apresentou um baixo número de pacientes com COVID mesmo com uma baixa taxa de isolamento social (SESPI, 2020; KOO JR, et al., 2020).

Levando em consideração os resultados encontrados, percebe-se que a população de 20-49 anos teve um destaque no que tange ao número de óbitos. Entretanto, a idade avançada se configura como fator de risco para a infecção pela COVID-19, visto que aumenta as chances de evolução para a Síndrome Respiratória Aguda Grave (SRAG). Porém, a faixa etária com maior número de óbitos foi a dos adultos jovens, esse fato pode ser esclarecido partindo da ótica de que o número de casos na faixa etária de 20-49 foi muito maior e por serem considerados mais vulneráveis, os idosos tem se prevenido mais (WU C, et al., 2020).

No que tange aos serviços de alta complexidade em uma das regiões mais afetadas, no estado da Bahia, em 2005 a 2014, observou-se um avanço na oferta de leitos da UTI adulto, tanto em serviços públicos quanto privados. Entretanto, apesar do crescimento em ambos os setores, o crescimento proporcional foi maior em serviços públicos.

Em relação a distribuição dos leitos na capital e nos demais municípios, também se notou aumento em ambos, todavia, Salvador ainda concentra a maior parte dos leitos da Bahia. A insuficiência de leitos de UTI, especialmente nos municípios do interior da Bahia, reflete falhas do Sistema Único de Saúde (SUS), especialmente no que tange ao princípio da integralidade (PASSOS JS, et al., 2018). 
A Atenção Primária à Saúde (APS) é de fundamental importância em situações emergenciais, entre elas o COVID-19, visto que influencia significativamente na diminuição da mortalidade e das desigualdades em saúde.

Algumas estratégias presentes na APS são essenciais no combate a pandemia, como o conhecimento do território, o acesso a comunidade, o vínculo presente entre usuários e equipe de saúde, a integralidade da assistência, o monitoramento das famílias vulneráveis e o acompanhamento dos casos suspeitos.

A APS é essencial na diminuição das iniquidades de saúde, em virtude do seu elevado grau de abrangência em território nacional e cobertura de parcelas da sociedade que estão expostas a excessivos riscos e por isso deve ser fortalecida e estruturada como uma das principais estratégias estabelecidas no combate à pandemia (DUNLOP C, et al., 2020; MENDONÇA MHM, et al., 2018; HONE T, et al., 2017; SARTI TD, et al., 2020).

A APS é porta de entrada para identificar e acolher todos os pacientes com suspeita de COVID-19, através de uma estratégia desenvolvida pelo Ministério da Saúde os pacientes são classificados conforme os sintomas, idade e doenças preexistentes e são encaminhados, se necessário, para os serviços de alta complexidade.

Os pacientes que não necessitam ser regulados, são orientados quanto ao isolamento domiciliar, com permanência contínua de contato direto com a equipe de saúde APS para controle e avaliação dos sintomas (BRASIL, $2020 \mathrm{c}$ ).

Tendo em vista a existência da fase de transmissão comunitária da COVID-19, é de suma importância que os serviços como os oferecidos pelas APS trabalhem com identificação sindrômica dos fatos. Acredita-se que com o fortalecimento da atenção hospitalar e estratégias de enfrentamento que se projetam para a comunidade, com ações governadas pelas APS contribuirão para um melhor controle da taxa de transmissão (BRASIL, $2020 \mathrm{c}$ ).

Considerando a eficácia das medidas de proteção contra a COVID-19 adotadas pelos estados e municípios, isolamento social, uso de máscaras e álcool gel, ainda se faz necessário garantir um aporte maior de leitos de Unidades de Terapia Intensiva (UTI) na região Nordeste. Atualmente, os leitos de UTI do Sistema Único de Saúde (SUS) corresponde a 57\% do número de leitos na região.

A Bahia, o segundo estado de maior prevalência em números de casos identificados nos resultados, contabiliza 1.152 leitos UTI SUS e 0,77 leitos UTI SUS por 10 mil habitantes, considerados ineficientes (MOTA E, et al., 2020).

Os achados deste estudo contribuem para divulgação da caracterização dessa pandemia na região nordeste do Brasil. Ressalta a importância da APS efetiva e de qualidade nos serviços junto à comunidade. Medidas como conscientização da população, modos de comportamento evitam a propagação desse vírus, prevenindo casos de morbidade e mortalidade. Evidenciou-se a necessidade de ampliação de leitos de UTI, unidade essencial para a assistência do paciente que apresente uma deterioração clínica, a exemplo de SARA, sendo necessário o aporte de respirador artificial para sua sobrevivência.

\section{CONCLUSÃO}

Conclui-se que no que tange ao perfil epidemiológico por COVID-19 no Nordeste, os maiores índices de casos confirmados e óbitos triunfaram nos estados mais populosos. Ao investigar a faixa etária, os casos confirmados predominaram na faixa de 20 a 49 anos e nos óbitos prevaleceu a faixa de 20 a 49 anos. Em relação ao sexo, observou-se uma preponderância dos casos confirmados no sexo feminino e nos óbitos prevaleceu o sexo masculino. É válido destacar que, por ser um estudo de acompanhamento, a elucidação e confirmação das conclusões presentes, só serão possíveis com o avanço dos conhecimentos acerca da temática. Entretanto, o estudo realizado é relevante para construção de novos dados, tendo em vista que podem possibilitar o desenvolvimento de medidas oportunas para o controle da COVID-19. 


\section{REFERÊNCIAS}

1. ASSOCIAÇÃO DE MEDICINA INTENSIVA BRASILEIRA (AMIB). São Paulo: Comunicado da AMIB sobre o avanço do COVID-19 e a necessidade de leitos em UTIS no futuro. Disponível em: http://www.somiti.org.br/arquivos/site/comunicacao/noticias/2020/covid-19/comunicado-da-amib-sobre-o-avanco-docovid-19-e-a-necessidade-de-leitos-em-utis-no-futuro.pdf. Acesso em: 29 mai. 2020.

2. BELASCO AGS, FONSECA CD. Coronavírus 2020. Rev Bras Enferm, 2020; 73(2): e2020n2.

3. BRASIL. Ministério da saúde. Atualização dos casos - Coronavírus. 2020 a. Disponível em: https://www.saude.gov.br/noticias/agencia-saude/46784-coronavirus-brasil-registra-52-995-casos-e-3-670-mortes. Acesso em: 24 abr. 2020.

4. BRASIL. Ministério da saúde. Secretarias Estaduais de Saúde. Coronavírus Brasil. 2020 b. Disponível em: https://covid.saude.gov.br/. Acesso em: 29 mai. 2020.

5. BRASIL. Ministério da saúde. Protocolo de Manejo Clínico do Coronavírus (COVID-19) na Atenção Primária à Saúde. 2020 c. Disponível em: https://www.saude.gov.br/images/pdf/2020/marco/20/20200318-ProtocoloManejo-ver002.pdf. Acesso em: 29 mai. 2020.

6. BRASIL. Ministério da Saúde. Secretaria Estadual de Saúde. Linha do Tempo Coronavírus. 2020. Disponível em: https://coronavirus.saude.gov.br/linha-do-tempo/\#fev2020. Acesso em: 15 jul. 2020.

7. CHEN N, et al. Epidemiological and clinical characteristics of 99 cases of 2019 novel coronavirus pneumonia in Wuhan, China: a descriptive study. Lancet, 2020; 395(10223):507-513.

8. CONSELHO FEDERAL DE ENFERMAGEM (COFEN). Comissão de Business Intelligence. Produto 2: Análise de dados dos profissionais de enfermagem existentes nos Conselhos Regionais. 2011. Disponível em: http://www.cofen.gov.br/wp-content/uploads/2012/03/pesquisaprofissionais.pdf. Acesso em: 29 mai. 2020.

9. CRODA J, et al. COVID-19 in Brazil: advantages of a socialized unified health system and preparation to contain cases. Rev. Soc. Bras. Med. Trop, 2020; (53): e20200167.

10. DIAS FLT, et al. Doenças respiratórias no Triângulo Mineiro: Análise epidemiológica e projetiva com a pandemia de COVID-19. J Health Biol Sci, 2020; 8(1):1-6.

11. DUNLOP $\mathrm{C}$, et al. The coronavirus outbreak: the central role of primary care in emergency preparedness and response. BJGP Open, 2020.

12. FREITAS ARR, et al. Análise da gravidade da pandemia de COVID-19. Epidemiol Serv Saude, 2020; 29(2): e2020119.

13. GORBALENYA AE, et al. Severe acute respiratory syndrome-related coronavirus: The species and its viruses - a statement of the Coronavirus Study Group of the International Committee on Taxonomy of Viruses. BioRvxiv preprint, 2020.

14. GUO YR, et al. The origin, transmission and clinical therapies on coronavirus disease 2019 (COVID-19) outbreakan update on the status. Mil Med Res, 2020; 7:11.

15. HONE T, et al. Association between expansion of primary healthcare and racial inequalities in mortality amenable to primary care in Brazil: a national longitudinal analysis. PLoS Med 2017; 14(5):e1002306.

16. KOO JR, et al. Interventions to mitigate early spread of SARS-CoV-2 in Singapore: a modelling study. Lancet, 2020; 20:678-88.

17. LANA RM, et al. Emergência do novo coronavírus (SARS-CoV-2) e o papel de uma vigilância nacional em saúde oportuna e efetiva. Cad Saude Pública, 2020; 36(3): e00019620.

18. LI Q, et al. Early Transmission dynamics in Wuhan - China, of novel coronavirus-infected pneumonia. N Engl J Med, 2020; 382(13):1199-1207.

19. LIMA DLF, et al. COVID-19 no estado do Ceará, Brasil: comportamentos e crenças na chegada da pandemia. Ciênc. saúde coletiva, 2020; 25(5): 1575-1586.

20. LU R, et al. Genomic characterization and epidemiology of 2019 novel coronavirus: implications for virus origins and receptor binding. Lancet, 2020; 395(10224):565-74.

21. MA C, et al. Incidence, clinical characteristics and prognostic factor of patients with COVID-19: a systematic review and meta-analysis. MedRxiv, 2020.

22. MENDONÇA MHM, et al. Atenção primária à saúde no Brasil: conceitos, práticas e pesquisa. Rio de Janeiro: FIOCRUZ, 2018; 612p.

23. MOTA E. Norte e Nordeste têm defasagem de leitos de UTI em relação às outras regiões do país. Universidade Federal da Bahia, 2020. Disponível em: https://coronavirus.ufba.br/norte-e-nordeste-tem-defasagem-de-leitos-de-utiem-relacao-outras-regioes-do-pais. Acesso em: 29 mai. 2020.

24. PASSOS JS, et al. Distribuição dos leitos de unidades de terapia intensiva adulto na Bahia. Enfermagem Brasil, 2018; 17(3): 266-272.

25. RENTE A, et al. Coronavírus e o Coração: Um Relato de Caso sobre a Evolução da COVID-19 Associado à Evolução Cardiológica. Arq Bras Cardiol, 2020.

26. ROTHAN HA, BYRAREDDY SN. The epidemiology and pathogenesis of coronavirus disease (COVID-19) outbreak. J Autoimmun, 2020; 109: 102433.

27. SCHATZMAYR HG. Viroses emergentes e reemergentes. Cad Saude Publica, 2001; 17(1):209-213.

28. SARTI TD, et al. Qual o papel da Atenção Primária à Saúde diante da pandemia provocada pela COVID-19? Epidemiol. Serv. Saúde, 2020; 29(2):e2020166.

29. SECRETARIA ESTADUAL DE SAÚDE DE PERNAMBUCO (SESPE). 2020. Disponível em: http://portal.saude.pe.gov.br/noticias/secretaria/novos-leitos-de-uti-comecam-receber-pacientes. Acesso em 29 mai.2020.

30. SECRETARIA ESTADUAL DE SAUDE DO PIAUÍ (SESPI). Coronavírus. 2020. Disponível em: http://coronavirus.pi.gov.br/. Acesso em: 29 mai. 2020.

31. WORLD HEALTH ORGANIZATION. Coronavirus disease (COVID-19) pandemic. 2020. Disponível em: https://www.who.int/emergencies/diseases/novel-coronavirus-2019. Acesso em: 24 mai. 2020.

32. WU C, et al. Risk Factors Associated With Acute Respiratory Distress Syndrome and Death in Patients With Coronavirus Disease 2019 Pneumonia in Wuhan, China. Jama Intern Med, 2020. 